ОБОСНОВАНИЕ. Диабетическая ретинопатия (ДР) - одно из самых частых офтальмологических осложнений сахарного диабета (СД), регистрируемое более чем у трети пациентов с СД. Выявление ранних маркеров ДР с помощью оптической когерентной томографии с функцией ангиографии (ОКТ-А) может способствовать совершенствованию диагностики и мониторинга заболевания.

ЦЕЛЬ. Изучить ранние функциональные и ОКТ-А-признаки ДР у пациентов с СД 1 типа (СД1) в отсутствие офтальмоскопических проявлений.

МЕТОДЫ. Были обследованы 68 человек (123 глаза), из них в группу СД вошли 40 пациентов (74 глаза, длительность

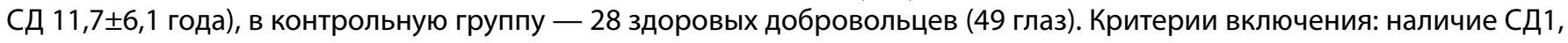
отсутствие офтальмоскопических признаков ДР; возраст от 18 до 45 лет. Всем участникам исследования был проведен стандартный офтальмологический осмотр, а также выполнена оценка остроты зрения в условиях низкой освещенности (ОЗУНО), 7-польная фоторегистрация глазного дна, ОКТ, ОКТ-А. Оцениваемые параметры: дефицит ОЗУНО, площадь фовеальной аваскулярной зоны (ФАЗ), индекс ациркулярности (ИА), сосудистая плотность (СП), скелетизированная плотность (СКП), индекс диаметра сосудов (ИДС), фрактальная размерность (ФР), толщина внутренних слоев сетчатки.

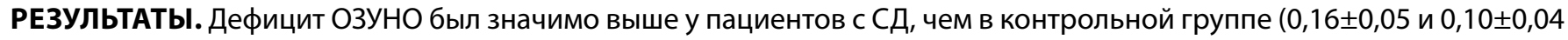
соответственно, $\mathrm{p}<0,0001)$. При оценке ОКТ-А-параметров мы обнаружили значимую разницу в показателях ИА

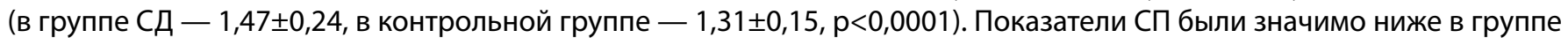

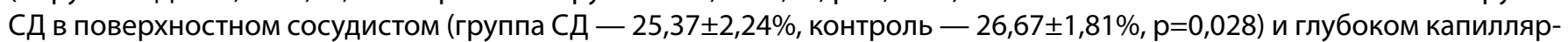

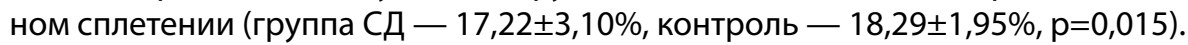

ЗАКЛЮчЕНИЕ. Полученные результаты свидетельствуют о наличии ранних функциональных и ОКТ-А-изменений на доклинической стадии ДР. Прогностическую значимость обнаруженных ОКТ-А-маркеров планируется изучить в ходе дальнейшего исследования.

КЛЮЧЕВЫЕ СЛОВА: сахарный диабет; диабетическая ретинопатия; оптическая когерентная томография; ретинальный кровоток

\title{
EARLY FUNCTIONAL AND MICROCIRCULATORY CHANGES IN PATIENTS WITH TYPE 1 DIABETES MELLITUS AND NO APPARENT DIABETIC RETINOPATHY
}

(c) Anna N. Stulova ${ }^{1 *}$, Nataliya S. Semenova1 , Anna V. Zheleznyakova², Vladimir S. Akopyan1', Dmitry V. Lipatov ${ }^{2,3}$, Marina V. Shestakova ${ }^{2}$

'Lomonosov Moscow State University, Moscow, Russia

${ }^{2}$ Endocrinology Research Center, Moscow, Russia

${ }^{3}$ Peoples Friendship University of Russia, Moscow, Russia

BACKGROUND: Diabetic retinopathy (DR) is the leading complication of diabetes mellitus (DM) which is reported to affect more than one-third of the global diabetic population. Optical coherence tomography angiography (OCT-A) can help to reveal early biomarkers of DR to improve current diagnostic approach.

AIMS: To evaluate early visual functions deficiency and OCT-A changes in patients with type 1 DM (T1DM) without clinical signs of DR.

MATERIALS AND METHODS. 74 eyes of 40 T1DM patients with no apparent DR and 49 eyes of 28 healthy volunteers were included in the study. The mean duration of DM was $11,7 \pm 6,1$ years. All participants underwent standard ophthalmological examination, low-luminance visual acuity (LLVA) assessment, 7-field fundus photography, OCT and OCT-A. We analyzed LLVA deficit as a marker of visual function. For OCT-A scans we evaluated foveal avascular zone (FAZ) area (mm2), acircularity 
index (Al), vessel density (VD), skeletonized density (SD) and vessel diameter index (VDI) in superficial vascular plexus (SVP), intermediate capillary plexus (ICP) and deep capillary plexus (DCP).

RESULTS: LLVA deficit was significantly higher in T1DM patients $(0,16 \pm 0,05$ vs $0,10 \pm 0,04, p<0,0001)$. We also detected a significant difference in Al level $(11,47 \pm 0,24$ vs $1,31 \pm 0,15, p<0,0001)$. VD was significantly lower in T1DM patients in SVP $(25,37 \pm 2,24 \%$, vs $26,67 \pm 1,81 \%, p=0,028)$ and $D C P(17,22 \pm 3,10 \%$ vs $18,29 \pm 1,95 \%, p=0,015)$

CONCLUSIONS: Our results demonstrate early visual functions deficiency and OCT-A changes at the preclinical stage of DR. The prognostic value of these findings will be studied during longitudinal observation.

KEYWORDS: diabetes mellitus; diabetic retinopathy; optical coherence tomography; retinal vessels

Диабетическая ретинопатия (ДР) - одно из самых частых офтальмологических осложнений сахарного диабета (СД), регистрируемое более чем у трети пациентов с СД. В 2016 г. распространенность ДР в РФ составила среди пациентов с СД 1 типа (СД1) - 38,3\%, СД 2 типа (СД2) 15,0\% [1]. Основными диагностическими методами, используемыми для выявления и мониторинга ДР, являются офтальмоскопия на фоне мидриаза и 7-польная регистрация глазного дна [2], предложенная рабочей группой Early Treatment Diabetic Retinopathy Study (ETDRS) в 1991 г. Золотым стандартом диагностики пролиферативной ДР остается флюоресцеиновая ангиография, однако на ранних стадиях осложнения ее применение нецелесообразно ввиду потенциальных рисков и высокой стоимости исследования. Оптическая когерентная томография с функцией ангиографии (ОКТ-А) расширила диагностический арсенал и позволила изучать микроциркуляторные изменения в сетчатке без введения контрастных веществ. Проведение ОКТ-А у пациентов с СД без видимых проявлений ДР показало наличие ранних признаков нарушения кровотока: изменений фовеальной аваскулярной зоны (ФАЗ), сосудистой плотности, появления неперфузируемых зон. В последнее время опубликовано большое число работ, посвященных этому вопросу, но только единичные исследования носят проспективный характер [3].

\section{ЦЕЛЬ}

Целью нашего исследования стало изучение ранних функциональных и ОКТ-А-признаков ДР у пациентов с СД1 в отсутствие офтальмоскопических проявлений.

\section{МЕТодЫ}

\section{Дизайн исследования}

Обсервационное двуцентровое одномоментное выборочное исследование.

\section{Критерии соответствия}

Критерии включения: возраст от 18 до 45 лет; наличие СД1, подтвержденного эндокринологом; отсутствие офтальмоскопических признаков ДР; наличие добровольного информированного согласия на участие в исследовании. Критерии исключения: миопия выше 6 диоптрий, помутнения оптических сред, другие заболевания органа зрения, хирургические вмешательства в анамнезе.

\section{Условия проведения}

Исследование проводилось на базе двух центров - кафедры офтальмологии ФФМ МГУ имени М.В. Ломоносова и отделения диабетической ретинопатии и офтальмохирургии ФГБУ «НМИЦ эндокринологии» Минздрава России.

Продолжительность исследования

Исследование проводилось с октября 2019 г. по март 2020 г.

\section{Описание медицинского вмешательства}

Всем участникам исследования был проведен стандартный офтальмологический осмотр (визометрия, тонометрия, офтальмоскопия на фоне мидриаза), а также выполнена оценка остроты зрения в условиях низкой освещенности (ОЗУНО), 7-польная фоторегистрация глазного дна, ОКТ, в том числе в режиме ангиографии. ОЗУНО оценивали по алгоритму Sunness J.S. и соавт. с помощью светофильтра нейтральной плотности с индексом затемнения $2.0 \log$ [4]. Дефицит ОЗУНО рассчитывали по формуле:

Дефицит ОЗУНО = Максимальная корригированная ОЗ (МКОЗ) - ОЗУНО.

Оценку ОЗ проводили с помощью десятичной таблицы Снеллена, для подсчета дефицита ОЗУНО и статистической обработки полученные значения переводили в эквиваленты по шкале LogMAR.

OKT-A осуществляли на приборах Swept Source DRI OCT Triton (Topcon, Япония) и SOCT Copernicus REVO (Optopol, Польша). Всем пациентам и здоровым добровольцам выполняли объемный скан макулярной зоны (М3) и сканирование M3 в режиме ангиографии $(3 \times 3$ мм и $6 \times 6$ мм). Программное обеспечение SOCT Copernicus REVO позволяет автоматически выделять сосудистые сплетения в соответствии с классификацией Campbell J.P. и соавт. [5]. При работе с DRI ОСT Triton сегментирование проводили вручную: границы поверхностного сосудистого сплетения (ПСС) - слоя нервных волокон сетчатки/слоя клеток ганглия (RNFL/GCL) - 23,4 мкм выше внутреннего сплетевидного слоя/внутреннего ядерного слоя (IPL/INL), промежуточного капиллярного сплетения (ПКС) - 23,4 мкм выше IPL/INL - 15,6 мкм ниже IPL/INL, глубокого капиллярного сплетения (ГКС) - 15,6 мкм ниже IPL/INL — 70,2 мкм ниже IPL/INL.

Обработку изображений и измерения проводили в программе Image J. Для вычисления площади и периметра ФАЗ использовали ОКТ-А-сканы поверхностного сплетения $3 \times 3$ мм. Границы ФАЗ обозначали вручную (рис. 1А). Для подсчета сосудистой плотности (СП) ОКТ-А-изображения преобразовывали в бинарную форму, после чего автоматически вычисляли отношение пикселей сосудистого рисунка к общему числу пикселей в кольцевых зонах диаметром 300 мкм (рис. 1Б). С по- 

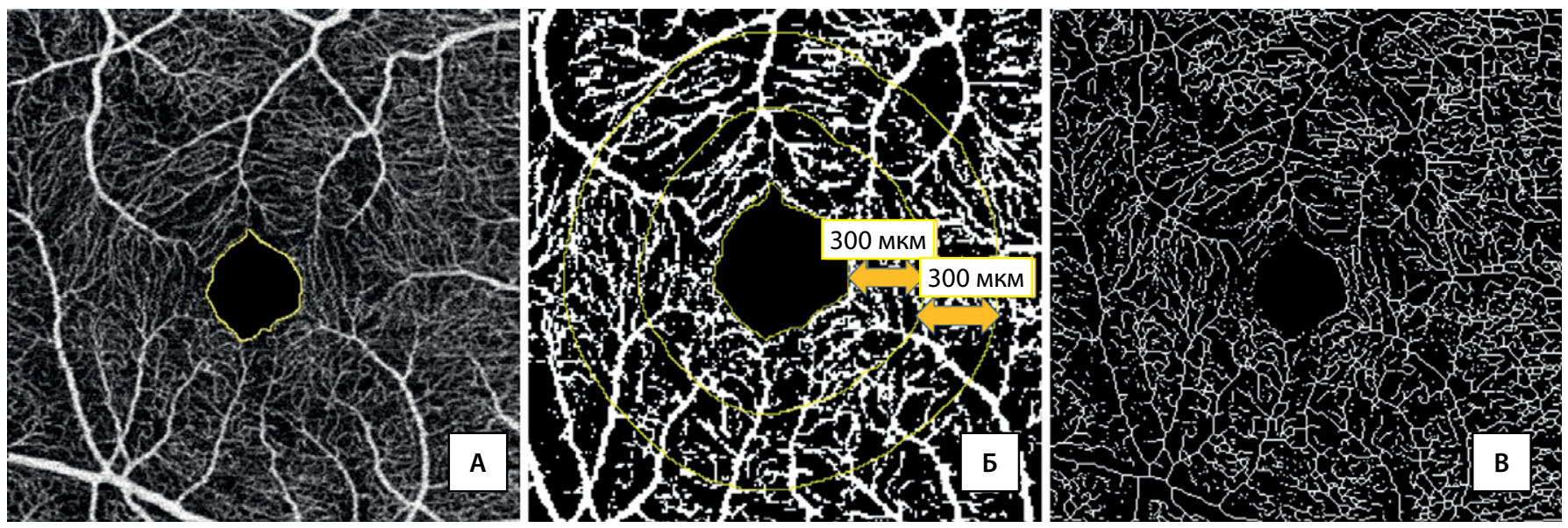

Рисунок 1. Этапы обработки изображения и подсчета количественных показателей. А - обозначение границ фовеальной аваскулярной зоны. Б - бинаризация изображения и выделение кольцевых зон для подсчета сосудистой плотности. В - скелетизация изображения для последующего вычисления скелетизированной плотности, индекса диаметра сосудов и фрактальной размерности.

мощью функции «skeletonize» выполняли скелетизацию бинарного изображения для подсчета скелетизированной плотности и фрактальной размерности (ФР) (рис. 1В).

В табл. 1 представлены перечень и краткое описание оцениваемых ангиографических параметров.

Наряду с количественными параметрами анализировали и качественные - наличие неперфузируемых зон (H3) и микроаневризм (МА). Для оценки использовали ОКТ-Асканы трех сплетений 3×3 мм и 6×6 мм. Два исследователя проводили независимый анализ ОКТ-А-сканов; Н3 и МА считали подтвержденными при совпадении двух мнений.

\section{Анализ в подгруппах}

В исследовании участвовали группа пациентов с СД1 без видимой ДР и контрольная группа (здоровые добровольцы).

\section{Этическая экспертиза}

Проведение исследования одобрено локальным этическим комитетом при ФГБУ «НМИЦ эндокринологии» Минздрава России (протокол №13 от 04.09.2019).

\section{Статистический анализ}

Принципы расчета размера выборки. Расчет размера выборки проводили на основании следующих параметров: статистическая мощность - 80\%, допустимая ошибка I рода - 5\%, средний уровень индекса ациркулярности (ИА) и его среднеквадратичное отклонение -

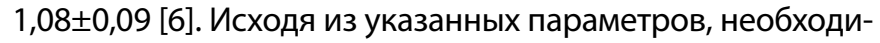
мый размер выборки составил 51.

Методы статистического анализа данных. Статистическую обработку проводили в программе IBM SPSS Statistics v.23.0. Описательная статистика представлена

Таблица 1. Количественные ангиографические параметры, оцениваемые в ходе исследования

\begin{tabular}{|c|c|c|}
\hline Название параметра & Алгоритм расчета & Краткое описание \\
\hline ФА $3, \mathrm{MM}^{2}$ & $\begin{array}{l}\text { Автоматическое вычисление площади } \\
\text { в пикселях, конвертирование в мм² }\end{array}$ & $\begin{array}{l}\text { Площадь ФАЗ }\left(\text { мм²}^{2}\right) \text {, границы } \\
\text { ФАЗ обозначены вручную }\end{array}$ \\
\hline ИА & 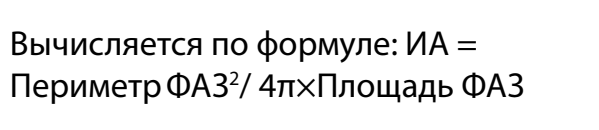 & $\begin{array}{l}\text { Отражает отклонения } \\
\text { формы ФАЗ от идеального } \\
\text { круга }\end{array}$ \\
\hline
\end{tabular}

Отношение числа пикселей сосудистого рисунка к общему числу пикселей в выбранной зоне. $\mathrm{C} \Pi, \%$ Две кольцевые зоны: СП0-300 (внутренняя граница - граница ФАЗ, внешняя граница 300 мкм от ФАЗ) и СП300-600 (внутренняя граница - 300 мкм от ФАЗ, внешняя 600 мКм от ФАЗ)

Позволяет получить представление о плотности капиллярного рисунка в выбранной зоне

Отношение числа пикселей скелетизированного сосудистого рисунка к общему числу пикселей изображения $3 \times 3$ мм

Отношение числа пикселей васкулярной сети бинарного изображения к соответствующему числу пикселей скелетизированного

Вычисляется автоматически с помощью плагина FractalCount
Выполняет ту же функцию, что и СП, но не зависит от диаметра сосудов

Относительная величина для характеристики диаметра сосудов

Характеризует степень сложности ветвления сосудов

Примечания: ФАЗ - фовеальная аваскулярная зона; ИА - индекс ациркулярности: СП - сосудистая плотность; СкП - скелетизированная плотность; ИДС - индекс диаметра сосудов; ФР - фрактальная размерность. 
в виде среднего значения \pm стандартное отклонение для показателей с нормальным распределением и медианы [25-й; 75-й процентили] для показателей с отклонением от нормального распределения. Нормальность распределения определяли в соответствии с критерием Колмогорова-Смирнова. Для сравнения параметров между группами использовали обобщенную линейную модель - обобщенные уравнения оценки для учета корреляции между парными глазами. Корреляцию между отдельными параметрами анализировали с помощью критерия Спирмена. Статистически значимыми считали различия при $\mathrm{p}<0,05$.

\section{РЕЗУЛЬТАТЬ}

\section{Объекты (участники) исследования}

Были обследованы 68 человек (123 глаза). В группу СД1 вошли 40 пациентов (14 мужчин, 26 женщин) (74 глаза; медиана возраста 25 лет [21,5; 31,5] с длительностью заболевания 10,0 [6,0; 16,3] лет, с уровнем гликированного гемоглобина $\left(\mathrm{HbA}_{1 c}\right) 7,1 \pm 1,1 \%$. В контрольную группу вошли - 28 здоровых добровольцев (10 мужчин и 18 женщин) (49 глаз; медиана возраста 25 лет [24; 26].

\section{Основные результаты исследования}

Сравнение функциональных показателей

При сравнении МКОЗ статистически значимых различий между группами обнаружено не было: группа СД1 - 1,0 [1,0; 1,0], контрольная группа - 1,0 [0,95; 1,0]. Дефицит ОЗУНО был значимо выше у пациентов с СД1,

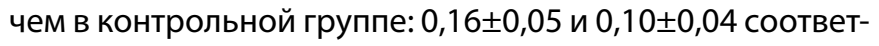
ственно, $\mathrm{p}<0,0001$ (рис. 2).

\section{сравнение структурных показателей}

Мы провели сравнительный анализ толщины внутренних слоев сетчатки - слоя нервных волокон сетчатки (СНВC), слоя ганглиозных клеток и их дендритов (СГК+) и слоя ганглиозных клеток с дендритами и аксонами (СГК++). Сравнения проводили в отдельных секторах в соответствии с сеткой ETDRS, статистически значимых различий между группами не выявлено. При оценке корреляции между дефицитом ОЗУНО и толщиной отдельных слоев мы зарегистрировали слабую отрицательную корреляцию с толщиной СНВС, СГК+

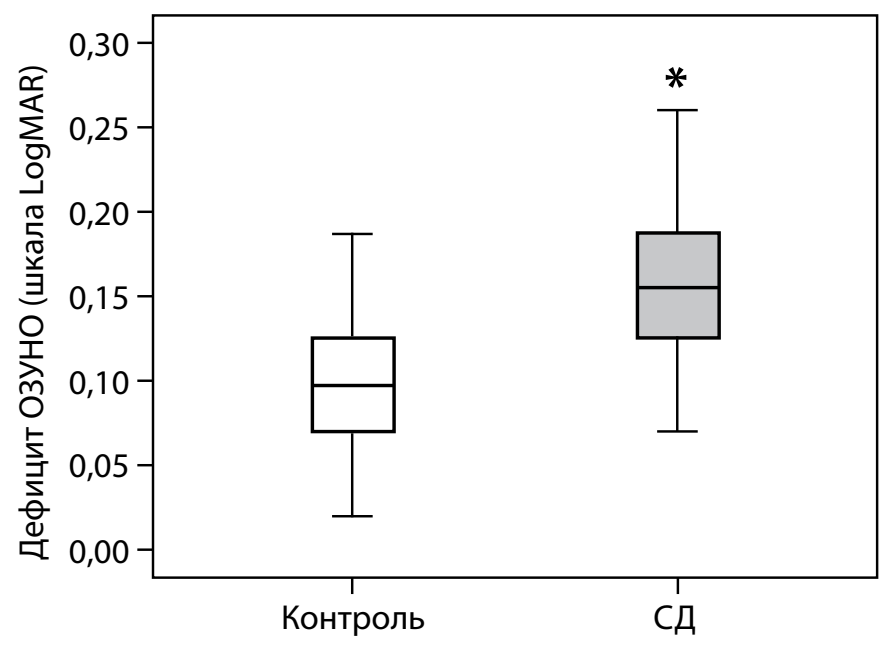

Рисунок 2. Сравнение дефицита остроты зрения в условиях низкой освещенности у пациентов с доклинической диабетической ретинопатией и в контрольной группе (*различия статистически значимы, $p<0,0001$, обобщенные линейные модели).

и СГК++ в отдельных секторах, результаты представлены схематично на рис. 3.

Анализ параметров ОКТ-А

В ходе предварительного сравнения значений параметров ОКТ-А-сканов, полученных на разных томографах, были выявлены статистически значимые различия для показателей плотности сосудистого рисунка и отсутствие различий для значений ФАЗ и ИА. Поэтому для статистической обработки ФАЗ и ИА был использован весь объем выборки, а для анализа параметров васкулярной плотности - только OKT-A-сканы, полученные на Swept Source томографе DRI OCT Triton. Кроме того, отдельные изображения были исключены из-за наличия артефактов. В табл. 2 представлены результаты статистического анализа ОКТ-А-параметров с указанием числа глаз.

В обеих исследуемых группах был обнаружен выраженный разброс значений площади ФАЗ, без значимых

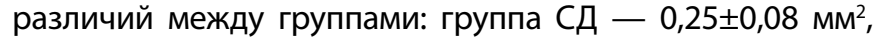

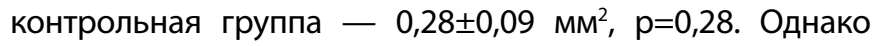
у пациентов с СД1 был значимо выше ИА: группа СД1 -

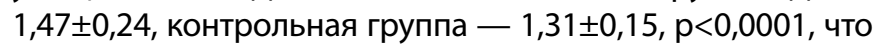
может указывать на изменение формы ФАЗ уже на ранних стадиях ДР.

\section{CHBC}

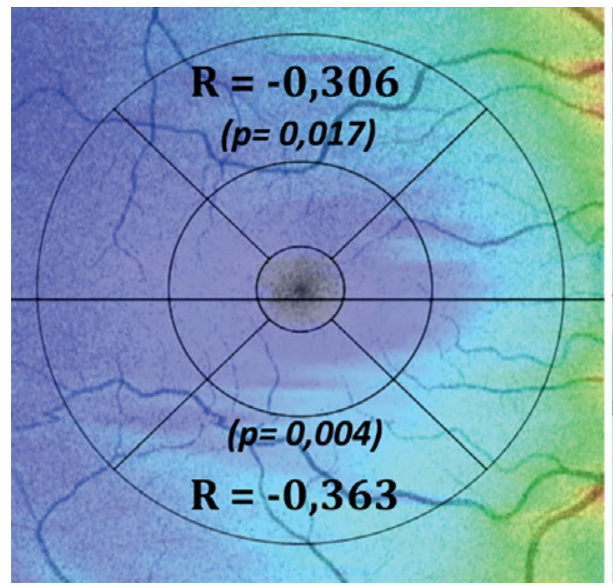

$\mathrm{C \Gamma K+}$

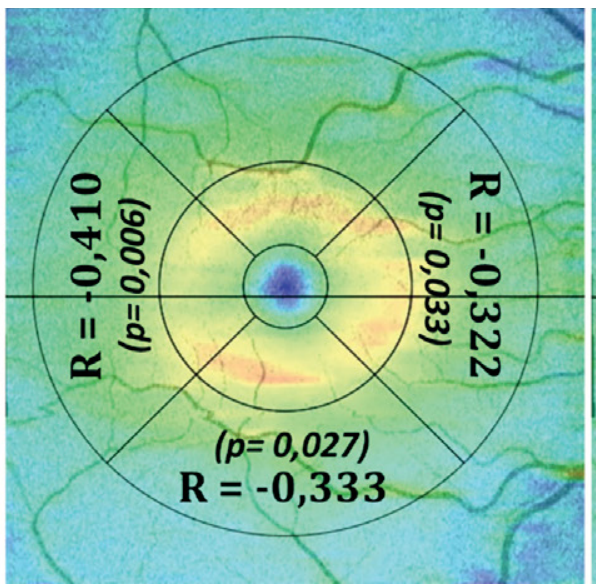

$\mathrm{C} \Gamma \mathrm{K}++$

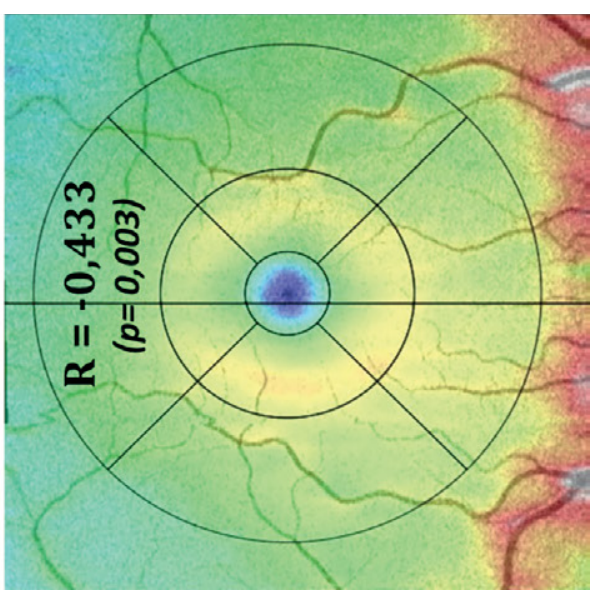

Рисунок 3. Схематичное изображение статистически значимых уровней корреляции толщины слоя нервных волокон сетчатки, слоя ганглиозных клеток и их дендритов (СГК+), слоя ганглиозных клеток с дендритами и аксонами (СГК++) в отдельных секторах и дефицита остроты зрения в условиях низкой освещенности ( $\mathrm{R}$ - коэффициент корреляции Спирмена, $\mathrm{p}$ - уровень значимости). 
Таблица 2. Результаты статистического анализа исследуемых ангиографических параметров

\begin{tabular}{|c|c|c|c|c|c|}
\hline \multirow[b]{2}{*}{ Параметр } & \multicolumn{2}{|c|}{ Контрольная группа } & \multicolumn{2}{|c|}{ Группа СД } & \multirow[b]{2}{*}{$\begin{array}{c}\text { Уровень } \\
\text { значимости }\end{array}$} \\
\hline & $\begin{array}{c}\text { среднее } \pm \\
\text { стандартное } \\
\text { отклонение или } \\
\text { медиана } \\
\text { [25-й; 75-й } \\
\text { процентили] }\end{array}$ & $\begin{array}{c}\text { Доверительный } \\
\text { интервал }\end{array}$ & $\begin{array}{c}\text { среднее } \pm \\
\text { стандартное } \\
\text { отклонение или } \\
\text { медиана } \\
\text { [25-й; 75-й } \\
\text { процентили] }\end{array}$ & $\begin{array}{c}\text { Доверительный } \\
\text { интервал }\end{array}$ & \\
\hline & \multicolumn{2}{|c|}{$n=41$} & \multicolumn{2}{|c|}{$n=66$} & \\
\hline ФА $3, \mathrm{Mm}^{2}$ & $0,25 \pm 0,08$ & $(0,23 ; 0,28)$ & $0,28 \pm 0,09$ & $(0,25 ; 0,30)$ & $p=0,28$ \\
\hline \multirow[t]{2}{*}{ ИA } & $1,31 \pm 0,15$ & $(1,26 ; 1,36)$ & $1,47 \pm 0,24$ & $(1,41 ; 1,53)$ & $p<0,0001 *$ \\
\hline & \multicolumn{2}{|c|}{$n=33$} & \multicolumn{2}{|c|}{$n=37$} & \\
\hline \multicolumn{6}{|l|}{ СПо-300 } \\
\hline пСС & $29,00 \pm 1,89$ & $(28,33 ; 29,67)$ & $27,90 \pm 2,29$ & $(27,13 ; 28,66)$ & $p=0,057$ \\
\hline ПКС & $29,00[28,0 ; 30,0]$ & $(28,74 ; 30,47)$ & $28,80[27,4 ; 30,2]$ & $(28,11 ; 30,17)$ & $p=0,694$ \\
\hline ГКС & $18,29 \pm 1,95$ & $(17,59 ; 18,98)$ & $17,22 \pm 3,10$ & $(16,76 ; 17,67)$ & $p=0,015^{*}$ \\
\hline \multicolumn{6}{|l|}{ СП300-600 } \\
\hline пСС & $26,67 \pm 1,81$ & $(26,03 ; 27,31)$ & $25,37 \pm 2,24$ & $(24,62 ; 26,11)$ & $p=0,028 *$ \\
\hline ПКС & $26,66[24,6 ; 28,1]$ & $(25,80 ; 27,96)$ & $26,05[24,4 ; 26,9]$ & $(25,36 ; 27,54)$ & $p=0,787$ \\
\hline ГКС & $14,41 \pm 1,02$ & $(14,05 ; 14,77)$ & $14,37 \pm 1,19$ & $(13,98 ; 14,77)$ & $p=0,876$ \\
\hline \multicolumn{6}{|l|}{ СкП } \\
\hline ПСС & $0,31[0,30 ; 0,32]$ & $(0,30 ; 0,31)$ & $0,15[0,14 ; 0,16]$ & $(0,15 ; 0,16)$ & $p<0,0001^{*}$ \\
\hline ПКС & $0,18[0,17 ; 0,19]$ & $(0,18 ; 0,19)$ & $0,18[0,17 ; 0,19]$ & $(0,18 ; 0,19)$ & $p=0,364$ \\
\hline ГКС & $0,098 \pm 0,006$ & $(0,096 ; 0,101)$ & $0,095 \pm 0,006$ & $(0,093 ; 0,097)$ & $p=0,015^{*}$ \\
\hline \multicolumn{6}{|l|}{ идс } \\
\hline псС & $1,97[1,92 ; 2,03]$ & $(1,94 ; 1,99)$ & $1,96[1,91 ; 2,01]$ & $(1,94 ; 1,98)$ & $p=0,450$ \\
\hline ПКС & $1,72 \pm 0,13$ & $(1,67 ; 1,76)$ & $1,69 \pm 0,04$ & $(1,67 ; 1,90)$ & $p=0,229$ \\
\hline ГКС & $1,78 \pm 0,03$ & $(1,77 ; 1,79)$ & $1,80 \pm 0,03$ & $(1,79 ; 1,81)$ & $p=0,036^{*}$ \\
\hline \multicolumn{6}{|l|}{ ФP } \\
\hline ПСС & $1,94[1,93 ; 1,95]$ & $(1,94 ; 1,94)$ & $1,94[1,93 ; 1,94]$ & $(1,93 ; 1,94)$ & $p=0,069$ \\
\hline ПКС & $1,95[1,95 ; 1,96]$ & $(1,95 ; 1,95)$ & $1,95[1,95 ; 1,96]$ & $(1,95 ; 1,95)$ & $p=0,849$ \\
\hline ГКС & $1,93[1,92 ; 1,93]$ & $(1,92 ; 1,93)$ & $1,92[1,92 ; 1,93]$ & $(1,92 ; 1,93)$ & $p=0,916$ \\
\hline
\end{tabular}

Примечания: ФАЗ - фовеальная аваскулярная зона; ИА - индекс ациркулярности; СП - сосудистая плотность; СКП - скелетизированная плотность; ИДС - индекс диаметра сосудов; ФР — фрактальная размерность; ПСС - поверхностное сосудистое сплетение; ПКС - промежуточное капиллярное сплетение; ГКС - глубокое капиллярное сплетение. Для оценки статистической значимости использовали обобщенные уравнения оценки, уровень статистической значимости - 0,05.

При сравнении результатов измерений сосудистой плотности (СП) в кольцевых зонах диаметром 300 мкм значимые различия были выявлены в поверхностном сосудистом сплетении (ПСС) и глубоком капиллярном сплетении (ГКС). Так, в поверхностном сплетении среднее зна-

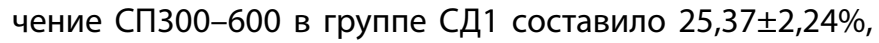

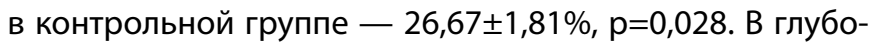
ком сплетении значимая разница была зарегистрирована в ближней к ФАЗ кольцевой зоне: СП0-300 в группе

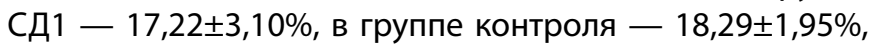
$\mathrm{p}=0,015$.

Наличие крупных сосудов в исследуемой зоне может искажать результаты анализа плотности васкулярной сети. Функция скелетизации позволяет устранить влияние диаметра сосудов. При оценке скелетизированная плотность (СКП) на всей площади ОКТ-А-скана $3 \times 3$ мм былизарегистрированызначимыеразличияв ПСС (вгруп-

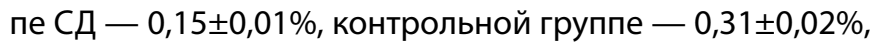

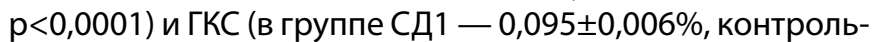
ной группе - 0,098 $0,006 \%, p=0,015)$. Кроме того, в ГКС

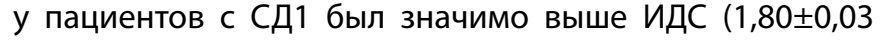
и $1,78 \pm 0,03, p=0,036)$.

При анализе ФР статистически значимых различий между группами обнаружено не было.

\section{Дополнительные результаты исследования}

Проведенный анализ корреляционной связи дефицита ОЗУНО и ангиографических параметров зарегистрировал отрицательную умеренную корреляцию между дефицитом ОЗУНО и СкП в ПСС ( $\mathrm{R}=-0,516 ; \mathrm{p}<0,0001)$, а также слабую корреляцию между дефицитом ОЗУНО и СП300-600 в ГКС (R=0,307; $p=0,045)$. Кроме того, дефицит ОЗУНО положительно коррелировал с ИДС в ПСС ( $R=-0,423 ; p=0,005)$.

При оценке качественных показателей в группе СД1 в 25,7\% глаз были выявлены Н3, регистрируемые преимущественно в ПКС. В 18,9\% глаз при детальном изучении ОКТ-А-сканов мы обнаружили признаки МА - в 2 глазах изолированно в ПСС, в 5 глазах - в ПКС, в 2 - в ГКС, в 2 - в ПСС и ПКС, в 3 - в ПКС и ГКС. На рис. 4 представлены примеры Н3 и МА. 


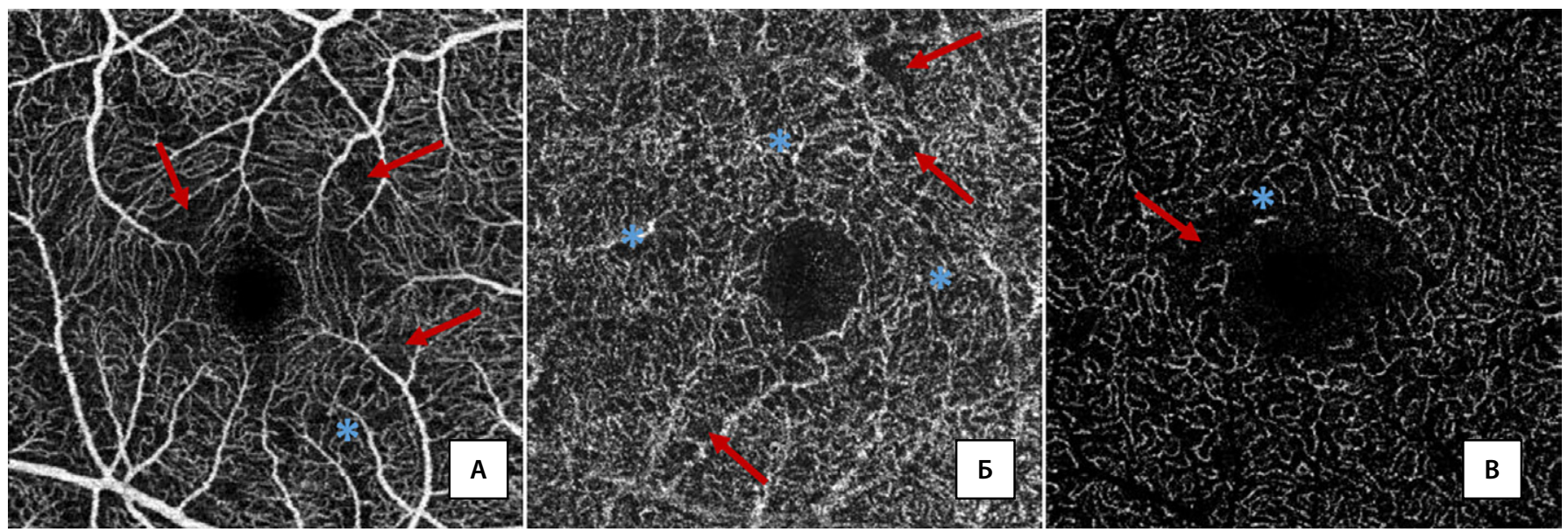

Рисунок 4. Примеры выявляемых неперфузируемых зон (Н3) и микроаневризм (МА) в поверхностном сосудистом сплетении - А, промежуточном капиллярном сплетении - Б, глубоком капиллярном сплетении - В. Красные стрелки указывают на Н3, голубые астериски - на МА

\section{ОБСУЖДЕНИЕ}

\section{Резюме основного результата исследования}

У пациентов с СД1 без офтальмоскопических признаков ДР было обнаружено статистически значимое снижение показателей СП и СкП на уровне ПСС и ГКС. Кроме того, на доклинической стадии ДР было выявлено повышение дефицита ОЗУНО и увеличение ациркулярности ФАЗ.

\section{Обсуждение основного результата исследования}

В нашем исследовании в качестве показателя зрительных функций мы использовали ОЗУНО. ОЗУНО была предложена Sunness J.S. и соавт. как предиктор снижения ОЗ у пациентов с сухой формой возрастной макулярной дегенерации (ВМД) [4]. Оценка дефицита ОЗУНО продемонстрировала свою воспроизводимость и прогностическую ценность у пациентов с ретинальными заболеваниями, затрагивающими M3 [7].

Полученные нами результаты указывают на увеличение дефицита ОЗУНО на доклинической стадии ДР, что можно рассматривать как ранний признак нейродегенерации. В 1990 г. Midena Е. и соавт. с помощью никтометрии продемонстрировали изменение макулярного ответа у 37,4\% пациентов с СД без видимой ДР [8]. Функциональные изменения на доклинической стадии ДР были позже подтверждены в исследованиях с применением статической периметрии, ритмической и мультифокальной электроретинографии (мфЭРГ) [9].

В ходе исследования мы не выявили значимой разницы между толщиной внутренних слоев сетчатки у пациентов с СД и здоровых добровольцев, однако была зарегистрирована значимая корреляция дефицита ОЗУНО и толщины СНВС, СГК+ и СГК++ в отдельных секторах. Данные литературы по этому вопросу противоречивы - снижение толщины СНВС и СГК зарегистрировано у пациентов с СД2 типа (СД2) и в смешанных группах [10]. В исследованиях, проведенных только с участием пациентов с СД1, реже описаны структурные изменения [11]. Кроме того, Vujosevic S. и соавт. продемонстрировали, что у пациентов с СД1 толщина СГК была значимо выше, чем у пациентов с СД2, с учетом поправки на возраст [12]. Авторы объясняют это более высоким риском развития периферической нейропатии и сердечно-сосудистых нарушений при СД2, что может обусловливать разные темпы нейродегенерации. На основании этого можно сделать вывод о необходимости проведения дальнейших исследований в изолированных группах с учетом возраста и типа СД.

Обнаруженное нами увеличение ациркулярности ФАЗ и отсутствие значимых различий площади ФАЗ между группами согласуется с результатами Inanc M. и соавт. и указывает на то, что ИА является более чувствительным параметром, чем площадь ФАЗ [6]. Ранее увеличение ФАЗ было описано у пациентов без офтальмоскопических признаков ДР, однако многие авторы отмечают большой разброс значений площади ФАЗ в норме и, как следствие, трудности в сравнении этого параметра на доклинической стадии $[13,14]$.

Результаты сравнения сосудистого рисунка свидетельствуют о наличии значимых различий СП и СкП в поверхностном и глубоком сплетениях. Большинство авторов описывают более выраженные изменения в ГКС, однако в настоящее время нет достаточных сведений о прогностической ценности показателей ОКТ-А в отдельных сплетениях [15]. Значимое увеличение ИДС в ГКС может указывать на ранние изменения диаметра сосудов вследствие нарушения механизмов ауторегуляции. Ранее Tang F. и соавт. зарегистрировали корреляцию ИДС с уровнем $\mathrm{HbA}_{1 с}$ и стадией ДР [16].

\section{ЗАКЛЮЧЕНИЕ}

Результаты проведенного исследования демонстрируют наличие значимых микроциркуляторных и функциональных изменений на доклинической стадии ДР. ОКТ-А позволяет регистрировать ранние нарушения капиллярного кровотока, проявляющиеся в изменении формы ФАЗ, снижении сосудистой плотности, появлении аваскулярных зон и МА. Рост дефицита ОЗУНО и его корреляция с ангиографическими показателями могут быть следствием сложного нейроваскулярного взаимодействия на ранних стадиях ДР. Обнаруженные изменения свидетельствуют о необходимости продолжения исследования в указанном направлении для установления прогностической ценности ОКТ-А-маркеров и разработки диагностических алгоритмов. 


\section{ДОПОЛНИТЕЛЬНАЯ ИНФОРМАЦИЯ}

Источник финансирования. Исследование выполнено в рамках государственного задания МГУ имени М.В. Ломоносова.

Конфликт интересов. Авторы декларируют отсутствие явных и потенциальных конфликтов интересов, связанных с публикацией настоящей статьи.
Участие авторов. Стулова А.Н. - проведение диагностических обследований; Железнякова А.В., Стулова А.Н. - формирование базы пациентов; Стулова А.Н., Семенова Н.С., Железнякова А.В. - анализ и интерпретация результатов исследования, написание текста статьи; Липатов Д.В., Акопян В.С., Шестакова М.В. - финальный анализ результатов и редактирование текста рукописи. Все авторы внесли существенный вклад в проведение исследования и подготовку статьи, прочли и одобрили финальную версию перед публикацией.

\section{СПИСОК ЛИТЕРАТУРЫ| REFERENCES}

1. Липатов Д.В., Викулова О.К., Железнякова А.В., и др. Эпидемиология диабетической ретинопатии в Российской Федерации по данным Федерального регистра пациентов с сахарным диабетом (2013-2016 гг.) // Сахарный диабет. - 2018. - T. 21. — №4. C. 230-240. [Lipatov DV, Vikulova OK, Zheleznyakova AV, et al. Trends in the epidemiology of diabetic retinopathy in Russian Federation according to the Federal Diabetes Register (2013-2016). Diabetes Mellitus. 2018;21(4):230-240. (In Russ.)]. doi: https://doi.org/10.14341/DM9797

2. Дедов И.И., Шестакова М.В., Майоров А.Ю., и др. Алгоритмы специализированной медицинской помощи больным сахарным диабетом: Клинические рекомендации (Вып. 9) // Сахарный диабет. - 2019. - T. 22. - №S1. - C.1-144. [Dedov II, Shestakova MV, Mayorov AYu, et al. Standards of specialized diabetes care. Diabetes Mellitus. 2019;22(S1):1-144 (In Russ.)]. doi: https://doi.org/10.14341/DM221S1

3. Forte R, Haulani H, Jürgens I. Quantative and qualitative analysis of the three capillary plexuses and choriocapillaris in patients with type 1 and type 2 diabetes mellitus without clinical signs of diabetic retinopathy: a prospective pilot study. Retina. 2020:40(2):333-344. doi: https://doi.org/10.1097/IAE.0000000000002376

4. Sunness JS, Rubin GS, Broman A, et al. Low Luminance Visual Dysfunction as a Predictor of Subsequent Visual Acuity Loss from Geographic Atrophy in Age-Related Macular Degeneration. Ophthalmology. 2008;115(9):1480-1488. doi: https://doi.org/10.1016/j.ophtha.2008.03.009

5. Campbell JP, Zhang M, Hwang TS, et al. Detailed vascular anatomy of the human retina by projection-resolved optical coherence tomography angiography. Sci Rep. 2017;7:1-11. doi: https://doi.org/10.1038/srep42201

6. Inanc M, Tekin K, Kiziltoprak H, et al. Changes in retinal microcirculation precede the clinical onset of diabetic retinopathy in children with type 1 diabetes mellitus. Am J Ophthalmol. 2019;207:37-44. doi: https://doi.org/10.1016/j.ajo.2019.04.011

7. Wood LJ, Jolly JK, Andrews CD, et al. Low-contrast visual acuity versus low-luminance visual acuity in choroideremia. Clin Exp Optom. 2021;104(1):90-94. doi: https://doi.org/10.1111/cxo.13087

8. Midena E, Segato T, Giuliano M, Zucchetto M. Macular recovery function (nyctometry) in diabetics without and with early retinopathy. Br J Ophthalmol. 1990;74(2):106-108. doi: https://doi.org/10.1136/bjo.74.2.106
9. Mermeklieva EA. Pattern electroretinography and retinal changes in patients with diabetes mellitus type 2. Neurophysiol Clin. 2019;49(3):209-215. doi: https://doi.org/10.1016/j.neucli.2019.04.002

10. Кулыбышева В.С., Ронзина И.А., Гамидов А.А., и др. Предикторы ранних изменений сетчатки при сахарном диабете // Офтальмология. — 2020. - Т. 17. — №1. C. 88-95. [Kulybysheva VS, Ronzina IA, Gamidov AA, et al. Predictors of early retinal changes in diabetes mellitus. Ophthalmology. 2020;17(1):88-95. (In Russ.)]. doi: https://doi.org/10.18008/1816-5095-2020-1-88-95

11. Pierro L, Iuliano L, Cicinelli MV, et al. Retinal neurovascular changes appear earlier in type 2 diabetic patients. Eur J Ophthalmol. 2017;27(3):346-351. doi: https://doi.org/10.5301/ejo.5000887

12. Vujosevic S, Midena E. Retinal Layers Changes in Human Preclinical and Early Clinical Diabetic Retinopathy Support Early Retinal Neuronal and Müller Cells Alterations. J Diabetes Res. 2013;2013:1-8. doi: https://doi.org/10.1155/2013/905058

13. De Carlo TE, Chin AT, Bonini FMA, et al. Detection of microvascular changes in eyes of patients with diabetes but not clinical diabetic retinopathy using optical coherence tomography angiography. Retina. 2015:35(11):2364-2370. doi: https://doi.org/10.1097/IAE.0000000000000882

14. Бурнашева М.А., Куликов А.Н., Мальцев Д.С. Персонализированный анализ фовеальной аваскулярной зоны с помощью оптической когерентной томографии ангиографии // Офтальмологические ведомости. 2017. - T. 10. — №4. - C. 32-40. [Burnasheva MA, Kulikov AN, Maltsev DS. Personalized analysis of foveal avascular zone with optical coherence tomography angiography. Ophthalmology Journal. 2017;10(4):32-40 (In Russ.)]. doi: https://doi.org/10.17816/OV10432-40

15. Scarinci F, Picconi F, Giorno P, et al. Deep capillary plexus impairment in patients with type 1 diabetes mellitus with no signs of diabetic retinopathy revealed using optical coherence tomography angiography. Acta Ophthalmol. 2018;96(2):264-265. doi: https://doi.org/10.1111/aos.13510

16. Tang FY, Ng DS, Lam A, et al. Determinants of quantitative optical coherence tomography angiography metrics in patients with diabetes. Sci Rep. 2017;7(1):1-10. doi: https://doi.org/10.1038/s41598-017-02767-0

\section{ИНФОРМАЦИЯ ОБ АВТОРАХ [AUTHORS INFO]}

*Стулова Анна Николаевна, аспирант [Anna N. Stulova, MD, PhD Student]; адрес: Российская Федерация, 119991, Москва, Ломоносовский пр-т, д. 27, к. 1 [27/1 Lomonosov Av., Moscow, Russian Federation, 119991]; ORCID: https://orcid.org/0000-0002-5121-803X; eLibrary SPIN: 5744-6530; e-mail: anna_stulova@mail.ru

Семенова Наталия Сергеевна, к.м.н., доцент [Nataliya S. Semenova, MD, PhD, associate professor]; ORCID: https://orcid.org/0000-0002-7928-5410; eLibrary SPIN: 5767-4851; e-mail: semenovans@gmail.com Железнякова Анна Викторовна, К.м.Н., С.H.с. [Anna V. Zheleznyakova, MD, PhD, senior research associate]; ORCID: https://orcid.org/0000-0002-9524-0124; Scopus ID: 55939275900; eLibrary SPIN: 8102-1779; Researcher ID: J-3455-2017; e-mail: azhelez@gmail.com

Акопян Владимир Сергеевич, д.м.н., професcop [Vladimir S. Akopyan, MD, PhD, Professor]; ORCID: https://orcid.org/0000-0003-0481-3642; eLibrary SPIN: 7185-9577; e-mail: akopyan_vs@yahoo.com

Липатов Дмитрий Валентинович, д.М.Н., професcop [Dmitry V. Lipatov, MD, PhD, Professor]; ORCID: http://orcid.org/0000-0002-2998-3392; eLibrary SPIN: 9601-3993; e-mail: glas1966@rambler.ru Шестакова Марина Владимировна, д.м.н., профессор, академик РАH [Marina V. Shestakova, MD, PhD, Professor]; ORCID: https://orcid.org/0000-0002-5057-127X; eLibrary SPIN: 7584-7015; e-mail: nephro@endocrincentr.ru 


\section{ЦИТИРОВАТЬ:}

Стулова А.Н., Семенова Н.С., Железнякова А.В., Акопян В.С., Липатов Д.В., Шестакова М.В. Ранние функциональные и микроциркуляторные нарушения у пациентов с сахарным диабетом 1 типа без видимой диабетической ретинопатии // Сахарный диабет. — 2021. — Т. 24. — №3. — C. 243-250. doi: https://doi.org/10.14341/DM12532

\section{TO CITE THIS ARTICLE:}

Stulova AN, Semenova NS, Zheleznyakova AV, Akopyan VS, Lipatov DV, Shestakova MV. Early functional and microcirculatory changes in patients with type 1 diabetes mellitus and no apparent diabetic retinopathy. Diabetes Mellitus. 2021;24(3):243-250. doi: https://doi.org/10.14341/DM12532 